\title{
Discurso, gramática e innovación metodológica en los tiempos del COVID... ¿o de la COVID?
}

\section{Discourse, grammar and methodological innovation in the times of COVID}

ELEna Carmona Yanes

Universidad de Sevilla

Departamento de Filología Francesa

elecarya@us.es

ORCID https://orcid.org/0000-0002-0427-2770

DOI: http://dx.doi.org/10.12795/9788447231003.074

Pp.: 1575-1598 


\section{Introducción}

El curso 2020/2021 se inició, para todos nosotros, estudiantes y docentes, con un clima atípico y colectivo de desasosiego personal e incertidumbre en todos los ámbitos, incluido el profesional. Nos encontramos repentinamente ante el reto de realizar un diseño didáctico con una información previa muy limitada sobre las circunstancias concretas en que se desarrollarían nuestras clases, y con la expectativa fundada de que tendríamos que trabajar con una incorporación acelerada de nuevos recursos tecnológicos (ahora indispensables para nuestra labor) y con un alumnado afectado, de un modo u otro, por la vivencia de una situación excepcional.

En este contexto, nos propusimos desarrollar un Ciclo de Mejora en el Aula (CIMA) que se aplicaría a la docencia de la asignatura obligatoria "Análisis gramatical y discursivo de textos franceses", del segundo curso del Grado en Estudios Franceses de la Facultad de Filología, que forma parte también del plan de estudios del Doble Grado en Estudios Franceses y Educación Primaria y cuya carga docente es de 6 créditos (3 prácticos y 3 teórico-prácticos). Las cifras de matriculación, que suelen superar los 80 e incluso los 90 estudiantes, se han mantenido similares este curso.

El hilo conductor de nuestro diseño inicial fue la intención de contribuir a minimizar todo sentimiento de incertidumbre poniendo el acento en los aspectos de nuestra tarea que son seguros y que no cambiarían respecto a otros años. Se proporcionó desde el principio información definitiva sobre la planificación de la docencia y sobre las actividades de evaluación, que no variarían fuera cual fuera el escenario sanitario en el que tuviéramos que desenvolvernos (esto es, la normalidad presencial y los

Ciclos de Mejora en el Aula (2020). Experiencias de Innovación Docente de la US Esta obra se distribuye con la licencia Creative Commons 
escenarios contingentes que la Universidad de Sevilla denominó A y B). Así, aprovechamos el aprendizaje obtenido a través del Programa FIDOP para crear una propuesta de trabajo y de evaluación compatible con todos los escenarios posibles. En ella nos servimos de las conclusiones y de los principios expuestos en nuestros dos artículos anteriores (Carmona Yanes 2018 y 2019) para implementar y diversificar una serie de mejoras metodológicas respecto a la impartición tradicional prototípica de una asignatura del área de la descripción y la teoría lingüísticas en francés, asumiendo el reto de trabajar con un grupo numeroso y en unas circunstancias excepcionales en las que puede complicarse el propósito de mantener al alumnado en un papel activo a pesar de las nuevas dificultades para la relación interpersonal, y de las posibles limitaciones técnicas, materiales e incluso psicológicas de cada individuo, que deberían poder recibir atención por parte del docente y de la institución.

Si en nuestros CIMA anteriores desarrollamos propuestas encaminadas a desautomatizar cognitivamente y contextualizar comunicativamente las actividades y los contenidos del análisis lingüístico, en esta ocasión nos centramos en la mejora de algunos otros aspectos, en concreto:

- El sistema de evaluación, con una modificación sustancial en el tipo de actividades que veníamos utilizando para la calificación oficial de la asignatura, que ahora se llevará a cabo integramente mediante actividades de evaluación continua que no requerirán la presencia física. Será nuestra apuesta fundamental para intentar transformar la incertidumbre en flexibilidad, y concentrar todo el trabajo extra de adaptación en la mejora metodológica permanente más allá de la coyuntura sanitaria.

Ciclos de Mejora en el Aula (2020). Experiencias de Innovación Docente de la US Esta obra se distribuye con la licencia Creative Commons 
- El desarrollo del componente investigativo en el aprendizaje, al que estarán directamente encaminadas las actividades de evaluación.

- El análisis del perfil psicológico y los estilos de aprendizaje de los estudiantes.

\section{Diseño previo del CIMA}

\section{Mapa de contenidos y problemas}

Además de la versatilidad respecto a una multiplicidad de escenarios posibles, uno de los principales cambios de diseño para este curso ha afectado a la ordenación temporal de los contenidos (Tabla 1). A lo largo de tres años de docencia y reflexión en el ámbito del análisis lingüístico, hemos ido justificando algunas modificaciones sobre la organización más habitual o tradicional de los contenidos, en coherencia con la concepción de la lingüística que poseo como investigadora y con los principios metodológicos que puse en práctica en los dos CIMA anteriores. Desde una perspectiva comunicativa y pragmática del estudio del lenguaje, parece más apropiado abordar el análisis de la lengua tomando como punto de partida el texto (o unidad máxima, de cuya construcción posee mayor consciencia el hablante y al que el interlocutor tiene un acceso más intuitivo) y no las unidades menores en las que se organiza la estructura de las lenguas, como se argumenta en Carmona Yanes (2018: 1571-1572). En consecuencia, en lo que se refiere a la selección de los contenidos principales, optamos también por dar mayor peso al análisis discursivo y textual, aun a sabiendas de que esto implicará que algunos contenidos morfológicos no podrían trabajarse con tanto detalle como otros años.

Ciclos de Mejora en el Aula (2020). Experiencias de Innovación Docente de la US Esta obra se distribuye con la licencia Creative Commons 
Con todo, dada la naturaleza descriptiva de esta materia, no se trata evidentemente de situar la atención a las formas gramaticales en un segundo plano, sino de explotar los procedimientos relacionados con la comprensión global o selectiva de un texto, típicos de los enfoques orientados a la acción de la enseñanza de lenguas extranjeras, para favorecer las destrezas de inferencia en las actividades de análisis lingüístico (cfr. Consejo de Europa 2001: 164-165). Por ello, muchas de las categorías discursivas se trabajan como contenidos procedimentales implícitos en la realización de las actividades de evaluación, lo que permite mantener una presencia suficiente de los contenidos conceptuales más relevantes del análisis morfosintáctico estructural. Ofrecemos igualmente una formulación renovada de los títulos de algunos de los epígrafes del proyecto docente, con la voluntad de tender un puente entre las nomenclaturas académicas más tradicionales y un enfoque más divulgativo y diverso, aunque igualmente riguroso, en cuanto a las elecciones terminológicas.

Tabla 1. Cambios en la ordenación temporal de los contenidos en el proyecto docente de la asignatura "Análisis gramatical y discursivo de textos franceses"

\section{Curso 2019-2020}

1. Parlons de linguistique: la communication publique, les textes et l'analyse grammaticale et discursive

1.1. Le discours et les débats sur la langue

1.2. Les sources de l'analyse linguistique

1.3. Terminologie: unités et relations linguistiques

\section{Curso 2020-2021}

\section{Parlons de linguistique: la} communication publique, les textes et l'analyse grammaticale et discursive

1.1. Le discours et les débats sur la langue.

1.2. Les sources et les outils de l'analyse linguistique. La recherche et les corpus linguistiques.

1.3. Terminologie et conventions. Unités et catégories linguistiques. 1.4. Les dimensions de l'analyse grammaticale: morphologie, syntaxe et sémantique.

Ciclos de Mejora en el Aula (2020). Experiencias de Innovación Docente de la US Esta obra se distribuye con la licencia Creative Commons 
2. Morphologie et lexicologie

2.1. Morphologie flexionnelle: la forme des mots

2.1.1. Flexion nominale et flexion adjectivale: le genre et le nombre 2.1.2. Flexion verbale

2.2. Morphologie dérivationnelle et lexicale: la formation des mots 2.2.1. Par dérivation: préfixation et suffixation

2.2.2. Par composition: composés et recomposés

\section{Morphosyntaxe de la phrase simple}

3.1. Formes et structures vs fonctions syntaxiques: terminologie

3.2.Le groupe du nom

3.3.Le groupe du verbe

3.4.Le groupe périphérique

3.5. Les différents types de phrase

\section{Syntaxe du texte et du}

discours: notions essentielles
2. Construire et déconstruire les textes : les relations syntaxiques. 2.1. La syntaxe du texte: cohérence, cohésion et segmentation du discours.

\subsection{Les groupes de mots.}

Morphosyntaxe de la phrase simple.

2.2.1. Fonctions syntaxiques vs rôles sémantiques.

2.2.2. Rection, subordination, accord et d'autres relations syntaxiques fondamentales.

2.2.3. La description et la terminologie des fonctions syntaxiques.

2.3. La représentation graphique des relations syntaxiques.

\section{La vie des mots : morphologie et lexicologie.}

3.1. Morphologie flexionnelle: la forme des mots.

3.1.1. Flexion nominale et flexion adjectivale: le genre et le nombre. 3.1.2. Flexion verbale.

3.2. Morphologie dérivationnelle et lexicale: la formation des mots. Dérivation, composition et d'autres procédés.

Los contenidos conceptuales se siguen relacionando, como el año pasado, de manera hipertextual o arbórea, como muestra el mapa de la figura 1: el bloque 1 se ocupa de nociones más generales que anticipan el marco conceptual de todos los aspectos más particulares de los bloques 2 y 3 . Transversalmente, en todos los bloques se trabajan los contenidos procedimentales y actitudinales que ya detallamos en Carmona Yanes (2019: 1247). Los objetivos de "hablar sobre la lengua" y de "expresarse como un lingüista" se siguen planteando como retos al inicio de la docencia.

Ciclos de Mejora en el Aula (2020). Experiencias de Innovación Docente de la US Esta obra se distribuye con la licencia Creative Commons 


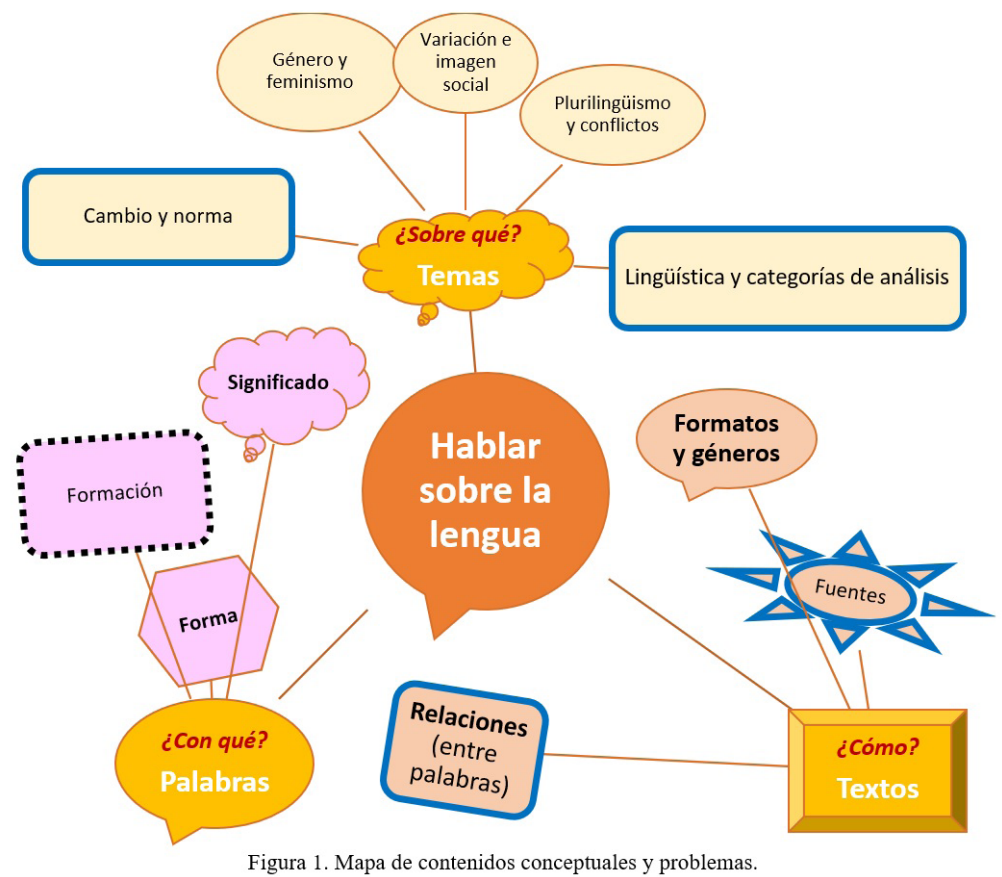

Por su parte, la figura 1 muestra una representación gráfica que aúna los vínculos conceptuales hipertextuales con el desarrollo temporal lineal del trabajo de la asignatura, lo que representa el esquema lógico que más se parecerá a la apariencia que tendrá la secuencia de actividades para los alumnos. Hemos utilizado para esta representación el recurso de la pirámide invertida que se emplea en periodismo: al igual que la información en un texto periodístico, tanto la organización general de nuestros contenidos como la organización dentro de cada bloque irá de los objetivos fundamentales e imprescindibles hasta el nivel de profundización al que cada "lector" desee llegar. En esta jerarquía se tendrá en cuenta la tradición escolar en la que presumiblemente se habrán formado nuestros alumnos. Así, una vez que hayamos constatado mediante los cuestionarios iniciales en qué punto de partida se sitúa el grupo (dando por sentado que no es la primera vez que trabajan contenidos de análisis lingüístico), nos moveremos Ciclos de Mejora en el Aula (2020). Experiencias de Innovación Docente de la US 
sucesivamente en secuencias de actividades que los llevarán a (1) aplicar las nociones ya conocidas a los casos prácticos más habituales, (2) aprender nociones más especializadas con las que resolver casos prácticos más complejos, (3) cuando sea posible, crear instrumentos de análisis propios para interpretar hechos inesperados o casos prácticos no prototípicos para la tradición escolar.

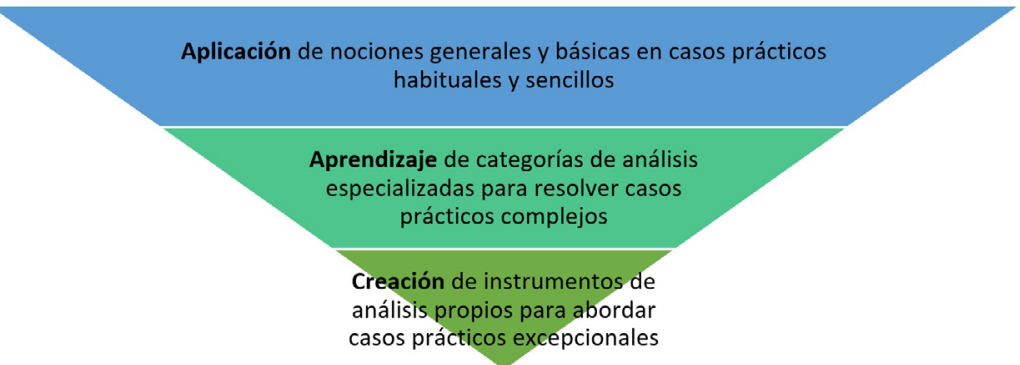

Figura 2. Organización conceptual/temporal de los contenidos

El resto de los cambios que se observan en la formulación de los contenidos en el proyecto docente pretenden dar carácter oficial a nuestra respuesta ante un problema detectado en cursos anteriores: la asimilación acrítica de una tradición terminológica escolar, que no tiene por qué ser la misma en todos los estudiantes, pero que estos tienden a seguir aplicando de manera automática en la etapa universitaria. Nuestra opción es un enfoque variacionista e historiográfico de la terminología, desde el que los alumnos puedan ir desarrollando progresivamente la conciencia del origen de las nociones más extendidas en la tradición escolar, el conocimiento de la existencia de distintas corrientes de estudio y la selección justificada y consciente de unas preferencias de uso consensuadas con el grupo, con capacidad para argumentar con rigor una elección diferente a la convenida si fuera necesario.

Por último, para reforzar el reto de expresarse como lingüistas a la hora de hablar de la lengua, se les propone 
otra aspiración, que es un método: ser filólogos y crecer con la lectura, para lo que utilizamos el siguiente lema desde el principio de la asignatura:

\section{"La lecture agrandit l'âme. "}

Voltaire

-R leRobert

Figura 3. "La lectura engrandece el alma"

\section{Modelo metodológico posible}

Mantenemos el modelo metodológico posible empleado nuestros CIMA anteriores, que se basa en la interacción entre las hipótesis de los alumnos y diferentes actividades de contraste a partir de preguntas de investigación o retos profesionales que generen la formulación de esas hipótesis. La exploración de las ideas previas puede dar lugar, a su vez, a nuevas preguntas de investigación que desencadenen de nuevo el circuito de aprendizaje. Otorgamos una relevancia especial al propósito de crear un entorno de aprendizaje crítico natural, en la línea de las propuestas de Bain (2007).

Consideramos que los principios básicos que resultan de este modelo metodológico no tenían por qué alterarse en las circunstancias actuales, y así lo justificamos en el plan de contingencia para el curso 2020-21 del proyecto docente:

[En el escenario (A) "Menor actividad académica presencial como consecuencia de medidas sanitarias de distanciamiento interpersonal que limiten el aforo permitido en las aulas"]. Se proporcionarán fichas de trabajo semanales o quincenales (en función del número de subgrupos

Ciclos de Mejora en el Aula (2020). Experiencias de Innovación Docente de la US Esta obra se distribuye con la licencia Creative Commons 
que establezca el Centro) que propondrán una planificación de las tareas para el período temporal en cuestión mediante actividades pertenecientes a las siguientes tipologías: (1) consulta y lectura guiadas de documentos; (2) participación en debates virtuales sobre las problemáticas trabajadas; (3) aplicación de categorías de análisis a casos prácticos. Las actividades del tipo (3) se pondrán en común presencialmente con cada subgrupo durante la sesión presencial que le sea asignada por el Centro 0 , en los casos en los que fuera necesario, mediante sesiones no presenciales síncronas. Las actividades de los tipos (1) y (2) se realizarán de manera asíncrona y los resultados serán supervisados por la profesora del mismo modo mediante los cauces virtuales específicos que se indiquen para cada caso. La distribución de actividades síncronas y asíncronas se establecerá en consonancia con las orientaciones metodológicas obtenidas mediante la formación docente proporcionada por el Programa de Formación e Innovación Docente del Profesorado (FIDOP) del Instituto de Ciencias de la Educación de la Universidad de Sevilla (https://sfep.us.es/ wsfep/sfep/formacion_docente.html), entre cuyos objetivos se destaca el "favorecer un proceso de cambio conceptual y metodológico que acerque al PDI de la Universidad, tanto el experimentado como al que se inicie en su carrera docente, a un modelo más innovador y acorde con los nuevos presupuestos emanados de las investigaciones que sobre educación, didáctica de las diversas disciplinas, psicología del aprendizaje, epistemología, neurociencia, etc. han venido desarrollándose en los últimos años". De estos planteamientos se derivan enfoques metodológicos alternativos a un modelo transmisivo tradicional, en los que se sitúa al discente en el centro del proceso de enseñanza-aprendizaje y se otorga al docente el rol de guía en ese proceso. Queda justificada, en consecuencia, la ausencia de necesidad prioritaria de difusión síncrona de las sesiones presenciales, ya que estas no consistirán sustancialmente en la exposición oral de información por parte del docente, y dado que las pruebas de evaluación no consistirán en un único documento de examen normalizado, sino en un dossier personalizado en el que cada alumno podrá demostrar la consecución de los objetivos globales centrándose en los contenidos específicos que sean más pertinentes para su proyecto individual.

Con esta redacción del proyecto docente, que entregamos semanas antes de conocer en qué circunstancias concretas se desarrollaría la docencia, intentábamos adelantarnos a los riesgos que podía correr nuestro modelo metodológico en un escenario semipresencial o híbrido, en el que podian presentarse algunos inconvenientes para el trabajo unificado del grupo en cualquier formato de sesión que no fuera una clase magistral, creándose, además, una no deseable dependencia constante de los recursos tecnológicos. Así, y como resultado de las reflexiones llevadas a 
cabo durante las reuniones con los compañeros de la REFID de mi equipo de Filología, me propuse desarrollar un planteamiento próximo al del aula invertida. En él, las sesiones presenciales podrían tener una concepción cercana a la de las tutorías: los estudiantes presentes podrian informar del progreso de sus proyectos y realizar consultas, mientras que los ausentes estarían avanzando en los suyos. Se podría utilizar, además, una parte de las seis horas de tutorías semanales para ofrecer tutorías colectivas en Blackboard Learn Collaborate en las que, si fuera necesario, se realizarían sesiones de puesta en común de resultados de actividades con el grupo completo o con la parte que deseara asistir.

En todo caso, de la adopción de este modelo metodológico y de la previsión de que su aplicación pudiera tener que llevarse a cabo en escenarios diversos se derivó el empleo sistemático de los recursos descritos a continuación, que eran compatibles con la presencialidad y con la no presencialidad, así como con el trabajo síncrono o asíncrono:

1) Los planes semanales o quincenales de trabajo recomendados.

2) Una carpeta de lecturas de consulta obligatoria habilitada en el espacio de Enseñanza Virtual. Este curso se ha incrementado el número de documentos con los objetivos de paliar posibles dificultades de acceso a los fondos de la biblioteca y de diversificar las fuentes accesibles en consonancia con los nuevos requisitos de la evaluación, en la que cobra un mayor peso la práctica investigativa sobre la de retención de datos.

3) Una guía bibliográfica que describe las referencias recogidas en el proyecto docente en un formato menos administrativo y más didáctico, aconsejando para qué fines puede ser más adecuado cada recurso bibliográfico. 
4) Las hojas o fichas de trabajo para realizar por escrito, en la línea del planteamiento para dar clase con la boca cerrada (Finkel 2008), que incluyen como elementos fundamentales un texto para analizar y una última actividad que deriva en la entrega de un pequeño resultado que la profesora puede revisar diaria o semanalmente, y que se concebirán como actividades auxiliares para la consecución de los objetivos de las actividades evaluables.

5) Las herramientas tecnológicas proporcionadas por la Universidad de Sevilla: Blackboard Learn Collaborate y los Debates de la plataforma de Enseñanza virtual.

6) Otros recursos informáticos para la interacción con grandes grupos, como Kahoot o Poll everywhere, que pueden utilizarse tanto en el aula como a distancia.

7) Un perfil de Twitter (@BavardagesLing), en el que llevamos más de un año difundiendo informaciones e ideas de interés para la materia (por medio de hashtags como \#viedesmots, \#maisquelgenre o \#normevaetvient), pero con la opción de gestionar también una serie de debates a través de la plataforma de Enseñanza Virtual, para que también el alumnado que no usa Twitter pueda publicar reacciones, comentarios, preguntas y sugerencias de lectura.

\section{Secuencia de actividades}

En el momento del diseño previo de la asignatura, no contábamos, por tanto, con información definitiva sobre cómo se iban a desarrollar exactamente las sesiones de clase en la Facultad de Filología, donde habitualmente las asignaturas de 6 créditos se imparten en tres sesiones semanales de 80 minutos. Cuando finalmente se nos comunicó que el curso se iniciaría en el escenario contingente $A$, y que el sistema semipresencial se organizaría mediante tres subgrupos que rotarían semanalmente, nos pareció apropiado prescindir de la semana cronológica como

Ciclos de Mejora en el Aula (2020). Experiencias de Innovación Docente de la US Esta obra se distribuye con la licencia Creative Commons 
unidad de referencia para la secuenciación. Entre otros motivos, porque, al haber semanas con días festivos, el tiempo presencial que se compartiría con cada subgrupo no sería equivalente, por lo que no podíamos proponer tres grupos de tareas, uno para trabajar presencialmente y otros dos no presencialmente, como era nuestra intención en un principio.

Creamos, así, siete micro-unidades de actividades, cada una de las cuales se compone de un número variable de sesiones (en función de criterios didácticos) y se publica anticipadamente a modo de plan de trabajo recomendado para los estudiantes, con indicaciones sobre las lecturas que sería conveniente realizar durante ese período, propuestas de fichas de actividades auxiliares para la consecución de los objetivos (cuyos resultados pueden corregirse o ponerse en común en las sesiones presenciales o en tutorías telemáticas) y entregas intermedias de tareas y documentos relacionados con los proyectos evaluables. Las tablas 2 y 3 son muestras del formato de fichas mediante el que se ha presentado esta planificación a los estudiantes.

Tabla 2. Ficha del plan de trabajo recomendado para la micro-unidad 2

\begin{tabular}{|c|c|c|}
\hline $\begin{array}{l}\text { Micro-unidad 2: La langue des } \\
\text { linguistes }\end{array}$ & № sesiones: 5 & Período: $26,27,28$ oct., $3-4$ nov. \\
\hline $\begin{array}{l}\text { Puntos del proyecto docente en } \\
\text { los que concentrarse }\end{array}$ & \multicolumn{2}{|c|}{$\begin{array}{l}\text { 1.3. Terminologie et conventions. Unités et catégories linguistiques. } \\
\text { 1.4. Les dimensions de l'analyse grammaticale : morphologie, syntaxe et } \\
\text { sémantique. }\end{array}$} \\
\hline $\begin{array}{l}\text { Lectura(s) recomendadas para el } \\
\text { período }\end{array}$ & \multicolumn{2}{|c|}{$\begin{array}{ll} & \text { Document } 1.3 \text { (ev.us.es/Contenido/Bloque 1) } \\
\square & \text { Document } 1.4 \text { (ev.us.es/Contenido/Bloque 1) }\end{array}$} \\
\hline $\begin{array}{l}\text { Objetivos principales de la micro- } \\
\text { unidad }\end{array}$ & \multicolumn{2}{|c|}{$\begin{array}{l}\checkmark \quad \begin{array}{l}\text { Conocer los principales términos de las nociones más generales del } \\
\text { análisis lingüistico en francés (unidades lingüísticas, clases de } \\
\text { palabras, ramas de la lingüística). }\end{array} \\
\checkmark \quad \text { Conocer otras convenciones gráficas y discursivas del discurso } \\
\text { metalingǘístico académico. } \\
\checkmark \quad \begin{array}{l}\text { Poder clasificar los elementos analizables como morfológicos, } \\
\text { sintácticos o semánticos. }\end{array}\end{array}$} \\
\hline $\begin{array}{l}\text { Fichas de trabajo y actividades } \\
\text { auxiliares recomendadas }\end{array}$ & \multicolumn{2}{|c|}{ 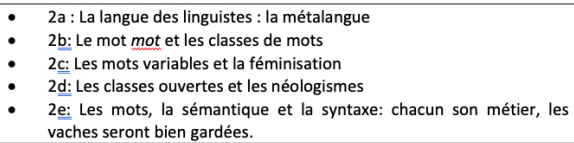 } \\
\hline Resultado a entregar & $\begin{array}{l}\text { Una lista justificada de } 3 \text { temas } \\
\text { interesantes para un texto } \\
\text { divulgativo, no académico, sobre } \\
\text { la lengua francesa }\end{array}$ & $\begin{array}{l}\text { Fecha de entrega: no más } \\
\text { tarde del } 28 \text { de octubre } \\
\text { (elecarya@us.es) (opcional) }\end{array}$ \\
\hline
\end{tabular}

Ciclos de Mejora en el Aula (2020). Experiencias de Innovación Docente de la US Esta obra se distribuye con la licencia Creative Commons 
Tabla 3. Ficha del plan de trabajo recomendado para la micro-unidad 3

\begin{tabular}{|c|c|c|}
\hline $\begin{array}{l}\text { Micro-unidad 3: Construire et } \\
\text { déconstruire les textes : les relations } \\
\text { syntaxiques }\end{array}$ & № sesiones: 6 & Período: 9, 10, 11, 16, 17, 18 nov. \\
\hline $\begin{array}{l}\text { Puntos del proyecto docente en } \\
\text { los que concentrarse }\end{array}$ & \multicolumn{2}{|c|}{$\begin{array}{l}\text { 2.1. La syntaxe du texte: cohérence, cohésion et segmentation du discours. } \\
\text { 2.2.1. Fonctions syntaxiques vs rôles sémantiques. } \\
\text { 2.2.2. Rection, subordination, accord et d'autres relations syntaxiques } \\
\text { fondamentales. }\end{array}$} \\
\hline $\begin{array}{l}\text { Lectura(s) recomendadas } \\
\text { para el período }\end{array}$ & \multicolumn{2}{|c|}{$\begin{array}{l}\square \text { Document 2.1. (ev.us.es/Contenido/Bloque 2) } \\
\square \text { Document 2.2, pages 1-3 (ev.us.es/Contenido/Bloque 2) }\end{array}$} \\
\hline $\begin{array}{l}\text { Objetivos principales de la micro- } \\
\text { unidad }\end{array}$ & \multicolumn{2}{|c|}{$\begin{array}{l}\checkmark \quad \begin{array}{l}\text { Tomar conciencia de los mecanismos que hacen de un conjunto de } \\
\text { frases un texto coherente y cohesionado. Poderlos identificar en un } \\
\text { texto bien o mal construido }\end{array} \\
\checkmark \quad \text { Aproximarse a la noción de conector } \\
\checkmark \quad \text { Profundizar en la delimitación entre las dimensiones morfológica, } \\
\text { sintáctica y semántica del análisis lingüístico } \\
\checkmark \quad \text { Entrar en contacto con nociones generales del análisis sintáctico }\end{array}$} \\
\hline $\begin{array}{l}\text { Fichas de trabajo y actividades } \\
\text { auxiliares recomendadas }\end{array}$ & \multicolumn{2}{|c|}{ 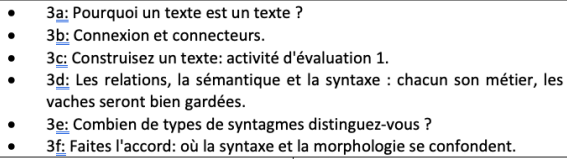 } \\
\hline Resultado a entregar & 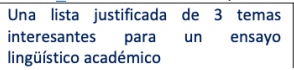 & $\begin{array}{l}\text { Fecha de entrega: no más tarde del } \\
30 \text { de noviembre (elecarya@us.es) } \\
\text { (opcional) }\end{array}$ \\
\hline
\end{tabular}

Seguimiento de la evolución de los modelos mentales de los estudiantes y de su aprendizaje

Como hemos indicado, el sistema de evaluación oficial de la asignatura se planteó desde un primer momento de manera que fuera compatible, sin ningún cambio, con todos los escenarios contingentes:

- La evaluación se llevará a cabo mediante la entrega de los resultados de tres proyectos que se desarrollarán a lo largo del período docente: (1) proyecto de divulgación del conocimiento lingüístico $(10 \%$ de la calificación final); (2) proyecto de redacción académica (30\% de la calificación final); (3) proyecto de casos prácticos de análisis gramatical y discursivo de textos franceses (60\% de la calificación final).

- La fecha de entrega del proyecto (1) se establecerá para el mes de noviembre de 2020; la fecha de entrega del proyecto (2) se establecerá para el mes de diciembre de 2020; la versión final del proyecto (3) deberá entregarse no más tarde de la fecha oficial establecida por la Secretaría de la Facultad para la prueba de evaluación.

Ciclos de Mejora en el Aula (2020). Experiencias de Innovación Docente de la US Esta obra se distribuye con la licencia Creative Commons Reconocimiento-NoComercial-SinObraDerivada Internacional (CC BY-NC-ND 4.0.) 
Para cada uno de estos proyectos se solicitaba, más concretamente, la entrega de las siguientes actividades:

1) Proyecto de divulgación del conocimiento lingüístico: la redacción de un artículo periodístico o de un texto literario que presenten contenidos lingüísticos a un público meta de perfil no académico. Con este proyecto se pretendía contribuir a vencer posibles resistencias iniciales o casos de escasa motivación hacia las cuestiones lingüísticas o gramaticales, y comenzar a enfocar el trabajo de un contenido procedimental fundamental, la distinción de registros en la redacción.

2) Proyecto de redacción académica: ensayo sobre un tema de relevancia para el ámbito del análisis gramatical y discursivo. Este proyecto les daría la oportunidad de profundizar en un tema concreto, de su propia elección, y de marcar en la redacción académica las distancias respecto a otros tipos de texto destinados a un grupo más amplio, como el que realizaron como proyecto 1.

3) Proyecto de casos prácticos de análisis gramatical y discursivo: recopilación de un corpus textual en el que puedan analizarse las principales categorías trabajadas a lo largo del curso. Esta propuesta invierte la costumbre tradicional de aportar los textos a los alumnos para que ellos apliquen las categorías de análisis estudiadas y evaluar si esta aplicación ha sido correcta. En este caso, valoraríamos si los contenidos conceptuales se han aprendido pidiendo que ellos busquen ejemplos significativos de cada categoría de análisis en cualquier tipo de producción lingüística a la que puedan tener acceso, tal como se procede en la investigación en esta área. Se trabajará específicamente la corrección en el tratamiento y la referencia a las fuentes, contenidos procedimentales y actitudinales de gran importancia. 
Además de estos instrumentos relacionados con la calificación oficial, este año hemos utilizado dos cuestionarios iniciales con fines diferentes:

- Un Cuestionario A, que usamos en la primera sesión para recabar información de tipo personal y psicológico que sea académicamente relevante.

- Un Cuestionario B, que empleamos durante la segunda sesión para detectar el estado previo de los estudiantes respecto a los contenidos principales de la asignatura. Gracias a estos datos, hemos contado con una base de comparación para el seguimiento del aprendizaje y la evolución de la consecución de objetivos relacionados con los contenidos conceptuales y procedimentales fundamentales.

\section{Aplicación del CIMA}

A la hora de iniciar la docencia, los datos recabados mediante el cuestionario A nos aportaron una información valiosa sobre las motivaciones previas de nuestros estudiantes, que en algún caso no se correspondían con nuestras expectativas. Destacaremos aquí las respuestas a dos de las preguntas planteadas. La primera versaba sobre sus hábitos lectores (Figura 4), y la segunda sobre la importancia concedida a una serie de componentes diversos y potencialmente motivadores de la asignatura (Tabla 4).

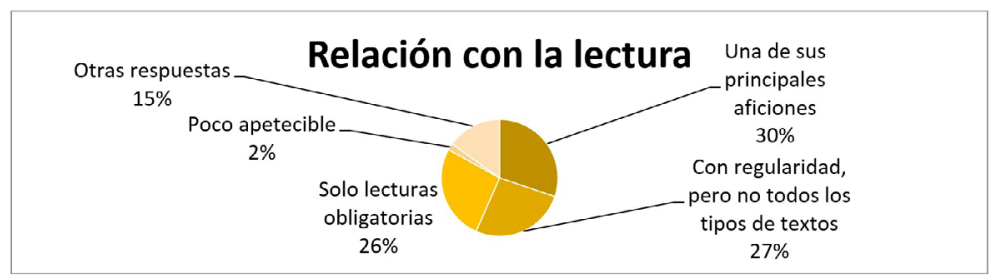

Figura 4. ¿Cómo es tu relación con la lectura?

Ciclos de Mejora en el Aula (2020). Experiencias de Innovación Docente de la US Esta obra se distribuye con la licencia Creative Commons 
Tabla 4. Puntúa de 0 a 10 la importancia que das a las siguientes cuestiones

(0: nada importante, 10: muy importante)

\begin{tabular}{|c|c|}
\hline I & 9,28 \\
\hline Quitarse la asignatura en febrero & 9,27 \\
\hline $\begin{array}{l}\text { Mejorar las competencias relacionadas con la comunicación } \\
\text { profesional }\end{array}$ & 9,19 \\
\hline $\begin{array}{l}\text { Mejorar las competencias relacionadas con la comprensión de } \\
\text { textos escritos complejos }\end{array}$ & 9,05 \\
\hline $\begin{array}{l}\text { Mejorar las competencias relacionadas con la comunicación } \\
\text { pública }\end{array}$ & 9,05 \\
\hline $\begin{array}{l}\text { Poder resolver casos prácticos con datos no previstos mediante la } \\
\text { observación y la reflexión }\end{array}$ & 8,65 \\
\hline $\begin{array}{l}\text { Saber que en francés la palabra mot es masculina y analyse } \\
\text { femenina }\end{array}$ & 8,62 \\
\hline Tener una nota superior a 7 en la asignatura, cueste lo que cueste & 8,21 \\
\hline Aprender muchos casos prácticos de análisis gramatical & 8,23 \\
\hline Aprender sobre la vida de las palabras & 8,15 \\
\hline $\begin{array}{l}\text { Conocer fuentes bibliográficas de utilidad para los estudios } \\
\text { lingüísticos }\end{array}$ & 8 \\
\hline $\begin{array}{l}\text { Seguir la actualidad sobre polémicas y debates lingüísticos en los } \\
\text { medios de comunicación }\end{array}$ & 7,42 \\
\hline $\begin{array}{l}\text { Conocer obras de divulgación científica del ámbito de la } \\
\text { lingüistica }\end{array}$ & 7,40 \\
\hline Tener una nota superior a 9 en la asignatura lo antes posible & 7,27 \\
\hline Superar rápidamente la asignatura, al menos con un 5 & 6,35 \\
\hline Comer caramelos & 3,07 \\
\hline
\end{tabular}

En primer lugar, estos datos nos permitieron constatar que nuestras impresiones de los cursos anteriores sobre los hábitos lectores de nuestros estudiantes de Filología se aproximaban a la situación manifestada por los integrantes de este grupo. Si bien un porcentaje cercano al $40 \%$ consideraba la lectura como una de sus principales aficiones (muchos de quienes seleccionaron la opción "Otras respuestas" manifestaban no leer más por falta de tiempo), una parte significativa del grupo se decantaba por la opción "Leo lo que me proponen en las asignaturas que

Ciclos de Mejora en el Aula (2020). Experiencias de Innovación Docente de la US Esta obra se distribuye con la licencia Creative Commons 
curso, pero en mi tiempo libre prefiero hacer otras muchas cosas", e incluso fue seleccionada en una ocasión la respuesta "Leer me resulta complicado, tedioso y poco apetecible". Si unimos a esto las reticencias indicadas en el $10 \%$ de las respuestas hacia tipos de textos como los periodísticos o los históricos, obtenemos la idea de que más de un tercio del grupo no tiene contacto regular con formas de comunicación escritas complejas, o no se aproxima a ellas con gusto o por motivación intrínseca. Sin embargo, la tabla 4 revela que las competencias relacionadas con la comunicación escrita y formal (en verde) son los componentes de la asignatura a los que conceden mayor importancia (por encima, incluso, de algunos objetivos relacionados con la evaluación sumativa, en naranja). Por el contrario, se valoran menos las competencias relacionadas con la investigación y la transferencia del conocimiento y la repercusión social de los temas lingüisticos (en azul), al menos por debajo de los aspectos relacionados explícitamente con contenidos prácticos concretos del análisis lingüístico (en amarillo).

Las motivaciones reflejadas en estos datos se vieron corroboradas cuando, al iniciarse el trabajo con el grupo, los estudiantes comenzaron a mostrar, en líneas generales, más dificultades no previstas para la preparación del primer proyecto de evaluación (un texto divulgativo, no académico) que para el segundo (un ensayo académico). La entrega del primer proyecto tras un mes y medio de docencia nos permitió comprobar, sin embargo, que se habían producido avances significativos en la capacidad de seleccionar temas potencialmente atractivos para el gran público y exponerlos en un formato y un registro adecuados.

Por lo demás, las primeras semanas de docencia estuvieron marcadas por la necesidad de afrontar nuestra labor cotidiana en un escenario nunca antes experimentado. Al margen del impacto psicológico y la incomodidad física 
de impartir sesiones presenciales con las debidas medidas sanitarias, se hizo muy complicado establecer relaciones interpersonales son un grupo de alumnos a los que no conocía previamente, y con los que debía interactuar simultáneamente a través de dos medios muy diferentes. A ello se unieron las numerosas incidencias técnicas acontecidas en el aula. Con frecuencia, los fallos de sonido y de conexión impidieron contactar con los subgrupos que se encontraban en casa. Por un lado, nuestro diseño didáctico se había anticipado a esas dificultades, y permitía realizar un trabajo guiado (por escrito) a quienes permanecían en sus hogares. Sin embargo, y pese a las indicaciones explícitas al respecto, costó bastante tiempo hacer que calara el mensaje de que el trabajo asíncrono con los materiales de la plataforma era tanto o más útil y necesario que las puestas en común orales. Los días en los que el micrófono del aula no estaba instalado o no funcionaba, un número importante de alumnos expresaba a través del chat su frustración y su inquietud por que pudiera estar ocurriendo "algo importante" en el aula. Fue una circunstancia que me causó desasosiego, ya que, aunque confiaba en que gran parte de los alumnos de los subgrupos ausentes estuvieran trabajando con los recursos escritos, no pude evitar, en varias ocasiones, desviar la atención del subgrupo presente para intentar resolver los problemas de comunicación en remoto.

Con la lamentablemente necesaria interrupción del sistema semipresencial el 9 de noviembre mejoró, no obstante, y en mi opinión, la dinámica de trabajo. Es cierto que ya había dispuesto de tiempo para llegar a conocer mejor al grupo, pero también que era más sencillo, desde la perspectiva del docente, atenderlos ahora a través de un único medio, aunque no fuera el más deseable. Las muestras de preocupación por parte de los estudiantes disminuyeron,

Ciclos de Mejora en el Aula (2020). Experiencias de Innovación Docente de la US Esta obra se distribuye con la licencia Creative Commons 
pasando a ser parecidas a las de cualquier año ordinario, y se redujo también mi nivel de ansiedad por la exposición al contagio, por baja que fuera, que había perjudicado mi docencia en las semanas anteriores. Poco a poco fui consiguiendo proporcionar un andamiaje cada vez más adecuado (al principio fue complicada la gestión del tiempo a la hora de la puesta en común de las actividades auxiliares suplementarias), explotar las muchas posibilidades para la interacción que ofrece Collaborate y ofrecer en las sesiones síncronas un verdadero espacio para la intervención abierta de un grupo numeroso, en el que un porcentaje significativo de asiduos terminó perdiendo el pudor para compartir de manera anticipada algunos de los resultados de sus proyectos de investigación. Las tutorías colectivas periódicas para reforzar esta participación fueron, probablemente, el recurso que recibió una acogida explícita más positiva.

Es necesario reconocer la dificultad que hemos experimentado para poder orientar hacia una manera de trabajar más autónoma a quienes requerían con frecuencia una supervisión muy personalizada que no era viable proporcionar a un grupo de casi cien personas. Encontrar el equilibrio entre el fomento de la autonomía y la autocrítica como contenidos actitudinales fundamentales y la sensación de que reciben atención y orientación por los medios oportunos sigue siendo para mí un reto principal.

\section{Evaluación del CIMA puesto en práctica.}

A falta de algunos datos sobre la evolución del aprendizaje que facilitarán los resultados completos de las actividades de evaluación, los ejercicios entregados a lo largo del período de docencia de la asignatura y los proyectos

Ciclos de Mejora en el Aula (2020). Experiencias de Innovación Docente de la US Esta obra se distribuye con la licencia Creative Commons 
ya calificados en el momento de redacción de este trabajo nos permiten apreciar que los resultados no ponen de manifiesto un retroceso respecto a los alcanzados en los cursos anteriores. Valoramos muy positivamente que, pese a las complicadas circunstancias, el porcentaje de actividades no presentadas no fuera alto, aunque sí ligeramente creciente (12\% para el primer proyecto y 18,5\% para el segundo). Las calificaciones parciales fueron igualmente positivas: el $32 \%$ de los proyectos presentados obtuvieron Sobresaliente, el 28,4\% Notable, el 19,8\% Aprobado y el porcentaje de suspensos fue del 19,8\%.

Entre los contenidos concretos cuya evolución hemos rastreado, podemos afirmar, con todas las cautelas que implica realizar comparaciones entre grupos de estudiantes diferentes, que los resultados de la progresión se muestran también muy similares a los de cursos anteriores. Ofrecemos a continuación las escaleras de dos de los contenidos a los que hemos dado mayor peso en la docencia: los procedimientos relacionados con la argumentación académica (Tabla 5) y el marcado tipográfico de los usos metalingüísticos (Tabla 6).

Ciclos de Mejora en el Aula (2020). Experiencias de Innovación Docente de la US Esta obra se distribuye con la licencia Creative Commons 
Tabla 5. Escalera 1

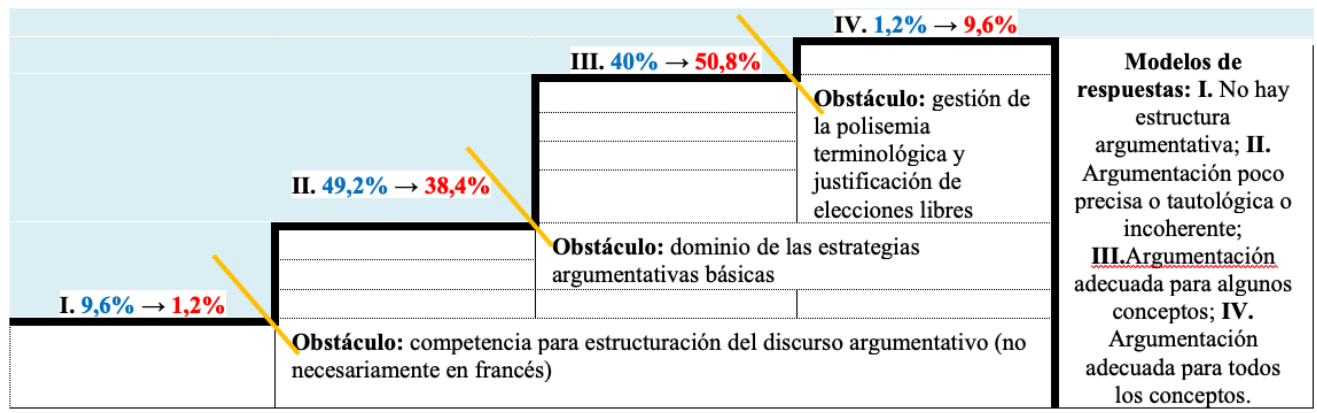

Tabla 6. Escalera 2

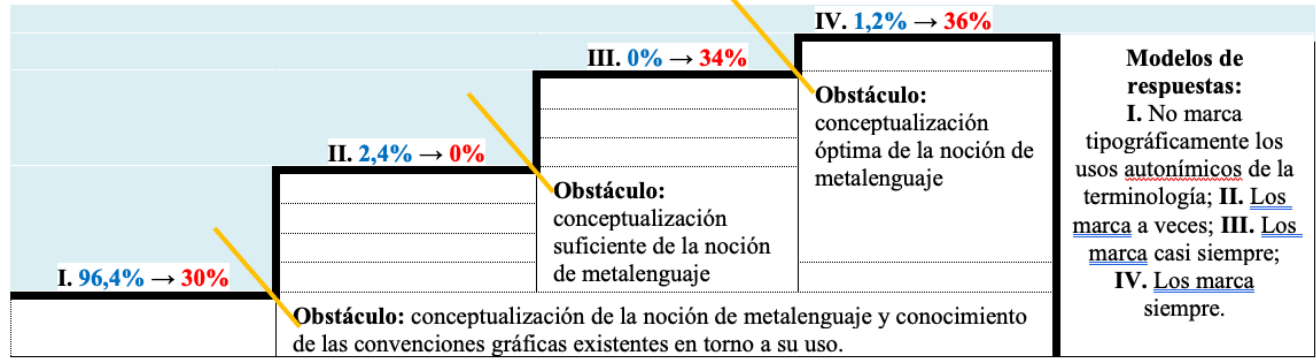

Los datos pueden contrastarse con los de nuestros CIMA de 2018-2019 y de 2019-2020 (Carmona Yanes 2018: 1583 y 2019: 1255), que reflejan una evolución similar. Más allá de las cifras, de los tres propósitos fundamentales que manifestábamos en la introducción, consideramos que es satisfactoria la realización de los dos primeros:

El planteamiento diseñado ha resultado idóneo para responder a las numerosas incidencias y cambios sobrevenidos a lo largo de este primer cuatrimestre (retraso del inicio de las clases en la Facultad de Filología, habituales fallos de conexión o audio entre el aula y los hogares e interrupción completa de la docencia presencial), y el sistema de evaluación elegido se ajusta sin dificultad a todos los escenarios, por lo que el plan de trabajo establecido desde el inicio ha podido mantenerse sin complicaciones

Ciclos de Mejora en el Aula (2020). Experiencias de Innovación Docente de la US Esta obra se distribuye con la licencia Creative Commons 
administrativas añadidas y permitiendo concentrar toda la energía en los aspectos metodológicos.

Igualmente, el componente investigativo de las actividades de evaluación se mostró adecuado para alcanzar los objetivos académicos que nos habríamos planteado en un curso ordinario por medio de una aproximación diferente, flexible y acorde con las circunstancias actuales, que aporta al alumnado la posibilidad de centrarse en los aspectos que puedan resultarle de mayor interés eligiendo entre distintas maneras posibles de organizarse temporalmente.

Señalaremos como aspecto susceptible de mejora el fomento de la autonomía psicológica para el trabajo asíncrono, pues, pese los logros conseguidos en este sentido, creemos que aún se podría hacer algo más para incrementar la sensación de confianza y seguridad en ellos mismos y en la orientación por escrito del docente durante las tareas individuales. El sentimiento de un grado importante de autosuficiencia resulta indispensable en un contexto en el que la ratio dificulta enormemente la implantación efectiva de metodologías que pongan de verdad al alumno en el centro del proceso de aprendizaje.

Concluiremos con una valoramos positiva de nuestra explotación de medios tecnológicos diversos para posibilitar la interacción con los estudiantes, lo que nos ha permitido llegar a familiarizarnos con sus modelos mentales incluso cuando sus rostros se nos mantenían ocultos. Blackboard Collaborate Ultra ha resultado, a mi juicio, ser un remedio coyuntural muy digno para la preservación de nuestro modelo metodológico en estos tiempos de pandemia. Hasta que sea posible recuperar la deseada normalidad, por supuesto preferible, las funcionalidades de esta plataforma (que ha presentado, además, un rendimiento técnico muy correcto) ofrecen para el trabajo en grupo opciones mucho más interesantes que la simple retransmisión de exposiciones magistrales. 
Palabras clave: Análisis gramatical y discursivo de textos franceses, Grado en Estudios Franceses, docencia universitaria, experimentación docente universitaria.

Keywords: Grammar and discursive analysis of French texts, Degree in French Studies, university teaching, university teaching experimentation.

\section{Referencias bibliográficas}

Bain, K. (2007). Lo que hacen los mejores profesores de universidad. Valencia: Universitat de Valencia.

Carmona Yanes, E. (2018). Propuesta de innovación docente para un análisis lingüistico activo, consciente y desautomatizado. Jornadas de Formación e Innovación Docente del Profesorado, 1, 1570-1587.

Carmona Yanes, E. (2019). Innovación y gramática: el metalenguaje contextualizado en el discurso de los medios de comunicación y las redes sociales. En R. Porlán y E. Navarro (Coords.), Ciclos de mejora en el aula, año 2019: experiencias de innovación docente de la Universidad de Sevilla (pp. 1241-1259). Sevilla: Editorial Universidad de Sevilla.

Consejo de Europa (2001). Marco común europeo de referencia para las lenguas: aprendizaje, enseñanza, evaluación. (traducido por el Instituto Cervantes 2002) Madrid: Anaya.

Finkel, D. (2008). Dar clase con la boca cerrada. Valencia: Universitat de Valencia.

Ciclos de Mejora en el Aula (2020). Experiencias de Innovación Docente de la US Esta obra se distribuye con la licencia Creative Commons 